Published in final edited form as:

J Inherit Metab Dis. 2012 November ; 35(6): 1037-1049. doi:10.1007/s10545-012-9477-y.

\title{
Diversity of approaches to classic galactosemia around the world: a comparison of diagnosis, intervention, and outcomes
}

\author{
Patricia P. Jumbo-Lucioni, \\ Department of Human Genetics, Emory University School of Medicine, Rm. 325.2 Whitehead \\ Bldg, 615 Michael St, Atlanta, GA 30322, USA
}

\section{Kathryn Garber,}

Department of Human Genetics, Emory University School of Medicine, Rm. 325.2 Whitehead

Bldg, 615 Michael St, Atlanta, GA 30322, USA

John Kiel,

Rollins School of Public Health, Emory University, Atlanta, GA, USA

Ivo Baric,

Department of Pediatrics, University Hospital Center, Zagreb, Croatia

Gerard T. Berry,

Division of Genetics, Children's Hospital Boston, Harvard Medical School, Boston, MA, USA

Annet Bosch,

Department of Pediatrics, Academic Medical Center, University of Amsterdam, Amsterdam, The Netherlands

Alberto Burlina,

Department of Pediatrics, Metabolic Unit, University Hospital, University of Padova, Padova, Italy

Ana Chiesa,

Centro de Investigaciones Endocrinológicas, CEDIE-CONICET, División Endocrinología, Hospital de Niños "Ricardo Gutiérrez", Buenos Aires, Argentina

Maria Luz Couce Pico,

Unidad de Trastornos Metabólicos, Hospital Clínico Universitario, Santiago de Compostela, Spain

Sylvia C. Estrada,

Institute of Human Genetics, National Institutes of Health, University of the Philippines Manila, Manila, Philippines

Howard Henderson,

Department of Chemical Pathology and School of Child and Adolescent Health, Red Cross

Children's Hospital, University of Cape Town, Cape Town, South Africa

Nancy Leslie,

Division of Human Genetics, Department of Pediatrics, Cincinnati Children's Hospital Medical Center and University of Cincinnati School of Medicine, Cincinnati, $\mathrm{OH}, \mathrm{USA}$

Nicola Longo,

\footnotetext{
(C) SSIEM and Springer 2012

jfridov@emory.edu.

Conflict of interest None.

Electronic supplementary material The online version of this article (doi:10.1007/s10545-012-9477-y) contains supplementary material, which is available to authorized users.
} 
Division of Medical Genetics, Department of Pediatrics, University of Utah, Salt Lake City, UT, USA

Andrew A. M. Morris, Willink Unit, Royal Manchester Children's Hospital, Manchester, UK

Carlett Ramirez-Farias, Genetics Nutrition Unit, National Institute of Pediatrics, Ministry of Health, Mexico City, Mexico

Susanne Schweitzer-Krantz, Children's Hospital, Evangelisches Krankenhaus, Düsseldorf, Germany

Catherine Lynn T. Silao, Institute of Human Genetics, National Institutes of Health, University of the Philippines Manila, Manila, Philippines

Marcela Vela-Amieva,

Genetics Nutrition Unit, National Institute of Pediatrics, Ministry of Health, Mexico City, Mexico

Susan Waisbren, and

Division of Genetics, Children's Hospital Boston, Harvard Medical School, Boston, MA, USA

Judith L. Fridovich-Keil

Department of Human Genetics, Emory University School of Medicine, Rm. 325.2 Whitehead

Bldg, 615 Michael St, Atlanta, GA 30322, USA

\section{Abstract}

Without intervention, classic galactosemia is a potentially fatal disorder in infancy. With the benefit of early diagnosis and dietary restriction of galactose, the acute sequelae of classic galactosemia can be prevented or reversed. However, despite early and lifelong dietary treatment, many galactosemic patients go on to experience serious long-term complications including cognitive disability, speech problems, neurological and/or movement disorders and, in girls and women, ovarian dysfunction. Further, there remains uncertainty surrounding what constitutes a 'best practice' for treating this disorder. To explore the extent and implications of this uncertainty, we conducted a small but global survey of healthcare providers who follow patients with classic galactosemia, seeking to compare established protocols for diagnosis, intervention, and follow-up, as well as the outcomes and outcome frequencies seen in the patient populations cared for by these providers. We received 13 survey responses representing five continents and 11 countries. Respondents underscored disparities in approaches to diagnosis, management and follow-up care. Notably, we saw no clear relationship between differing approaches to care and long-term outcomes in the populations studied. Negative outcomes occurred in the majority of cases regardless of when treatment was initiated, how tightly galactose intake was restricted, or how closely patients were monitored. We document here what is, to our knowledge, the first global comparison of healthcare approaches to classic galactosemia. These data reinforce the idea that there is currently no one best practice for treating patients with classic galactosemia, and underscore the need for more extensive and statistically powerful comparative studies to reveal potential positive or negative impacts of differing approaches.

\section{Introduction}

Classic galactosemia (OMIM 230400) is an autosomal recessive disorder that results from a profound defect in the enzyme galactose-1-phosphate uridylyltransferase (GALT, EC 2.7.7.12), leading to an impaired ability to metabolize galactose. The disease is characterized by potentially lethal acute symptoms in the neonatal period that can be prevented or reversed through the immediate and rigorous restriction of dietary galactose. While it is unquestioned 
that galactose withdrawal from the diet is crucial for avoidance or resolution of lifethreatening neonatal complications, dietary intervention alone does not eliminate long-term sequelae of the disorder. Unfortunately, and despite early and strict dietary restriction of galactose, a majority of patients reported in the literature grow to experience complications that can include cognitive disability, speech problems, neurological and/or movement disorders, and for girls and women, ovarian dysfunction (Bosch 2006; Fridovich-Keil and Walter 2008; Schweitzer-Krantz 2003; Waggoner et al 1990). Are these complications an inevitable feature of GALT impairment, or might they also reflect environmental influences?

Health care professionals and the families they serve face numerous diagnostic and treatment options and disparities surrounding classic galactosemia. Perhaps the most obvious is that in some populations affected babies are diagnosed pre-symptomatically by newborn screening, while in other populations affected infants are diagnosed only after clinical presentation. Might early acute disease predispose to long-term complications? Differences in the management of galactosemia also impact the rigor and persistence of dietary galactose restriction. Perhaps these differences, too, might influence long-term outcome. Finally, differences in monitoring or follow-up protocols might influence when danger signs or complications are first noted so that appropriate interventions can be initiated. Long-term outcome severity might reflect these differences as well.

Another factor that might contribute to differences in the perceived or reported severity of patient outcome is the local definition of classic galactosemia. The key is defining how much residual GALT activity a patient may have before they are no longer said to have "classic" galactosemia - but to have variant galactosemia instead. This is an important distinction because Duarte variant galactosemia (Carney et al 2009) is ten times as prevalent as true classic galactosemia in some populations, so that including even a fraction of Duarte patients, who are believed to be asymptomatic, in with the ranks of patients who have true classic galactosemia, could significantly alter the perceived long-term outcome profile. The population prevalence of non-Duarte GALT alleles that encode residual activity may also vary between populations.

We initiated this project to explore the potential impact of different diagnostic and management strategies for classic galactosemia currently practiced in different populations, seeking to learn from an ongoing "natural experiment." The work described here represents a first step toward that goal. Using surveys distributed to health care professionals who follow patients with classic galactosemia, we sought: (1) to compare and contrast established protocols for the diagnosis, intervention, and follow-up care of patients with classic galactosemia, (2) to compare and contrast patient outcomes and outcome frequencies in these different populations, and (3) to ask whether there is any clear relationship between stated approaches to diagnosis, intervention, or follow-up care and the outcomes experienced by the patient populations studied. Our results document diverse approaches to a single disease, but reveal no clear relationships between any of the healthcare parameters measured and long-term outcome severity.

\section{Methods}

We assembled a questionnaire survey (see Supplemental information) to collect information from healthcare providers who directly follow patients with classic galactosemia. This survey and the study design were submitted to the Emory University Institutional Review Board but were exempted from review. The survey was sent electronically to potential respondents identified from public sources, such as web pages of appropriate professional societies, corresponding author contact information from professional journal articles about 
galactosemia, and so forth. Some respondents were recommended by other respondents. Of greater than 30 contacts attempted, we received 13 completed survey responses representing the following locations: Argentina (Buenos Aires), Croatia (Zagreb), Germany (Düsseldorf), Italy (Padova), Mexico (Mexico City), the Netherlands (Amsterdam), the Philippines (Manila), South Africa (Cape Town), Spain (Santiago de Compostela), United Kingdom (Manchester), and USA (Salt Lake City, Boston, and Cincinnati). While broad, this collection of respondents nonetheless represents only a small fraction of countries and human population groups. Furthermore, we often had only one or two respondents from a given country, so it is impossible to know how uniform or disparate care and outcomes might be within each country. While informative, these data and our interpretations must therefore be considered an anecdotal first approach to a very complex problem.

Each respondent was asked to answer questions based on their own individual clinical practice. The survey consisted of 27 questions subdivided into the following categories: providers' and patients' characteristics, predominant health care system in the jurisdiction, diagnosis, early intervention practices, dietary considerations, puberty and teen years, longitudinal care, considerations for adults, and long-term outcomes. All the survey questions were open-ended to give respondents as much flexibility as possible in their crosssectional responses. Most of the data were collected between 2007 and 2008. In tabular format, providers were also asked to supply information on the relative frequencies of outcome issues among their patients (i.e., cognitive disability, behavioral or social problems, ovarian insufficiency in women, neurological problems, reduced bone density, visual impairments, speech difficulties, weight/height development, anemia, liver and/or kidney disease and vitamin D deficiency). Each respondent was asked to estimate each outcome frequency considering only those patients in their practice who were old enough and/or the appropriate gender to accurately assess each specific outcome. Ambiguous responses and/or outcomes, or responses for which fewer than three patients were evaluated, were excluded. Responses were compiled, compared, and analyzed for trends. Given the small size and anecdotal nature of the final sample set we did not apply any statistical methods.

\section{Results}

\section{Demographic characteristics of respondents and their patients}

The respondents were all health care professionals directly involved in the care of patients with classic galactosemia; many were pediatric endocrinologists, medical geneticists, and/or metabolic specialists with leading positions at their institutions. Our respondents saw a combined total of 234 patients and each managed an average of 20 patients per year (range=1-72). The patient racial makeup included Caucasian $(n=191)$, Hispanic $(n=29)$, African American ( $n=5)$, African $(n=1)$, and Asian $(n=8)$. The number of patients seen by each respondent, and the racial makeup of each group, are presented in Table 1.

Respondents estimated the disease frequency in their local populations at between 1:14,000 and 1:80,000 (mean 1:41,219).

Although there is no clear method by which we can discern and compare the numbers of infants "missed" by the different approaches to galactosemia diagnosis, it is interesting to point out that in the case of Mexico, providers estimated that more than $80 \%$ of infants were missed and therefore presumably lost due to incomplete or inadequate newborn screening. While half of the countries included in this survey relied mainly on a government-sponsored healthcare system for patient care (UK, Spain, Italy, Croatia, Germany and South Africa), the remaining countries displayed more complex healthcare system architectures with patients resorting to either government or private health insurance policies for their treatment and follow-up (USA, Mexico, Argentina, the Philippines, and the Netherlands). 


\section{Diagnostic approaches of the reporting jurisdictions}

Galactosemia was diagnosed by newborn screening for a majority of the patients of respondents from the Netherlands, US cities: Boston and Salt Lake City, Germany, the Philippines, and Spain, by clinical symptoms for a majority of the patients of respondents from Croatia and South Africa, and by a combination of both approaches for patients of respondents from the UK, Mexico, Italy, Argentina, and US city: Cincinnati. One respondent noted that although his country does not screen newborns for GALT deficiency, their screens for phenylketonuria aid in the ascertainment of galactosemic infants (Henderson et al 1988) because the blood spots from galactosemic infants often show elevated phenylalanine plus elevated tyrosine; infants with phenylketonuria show elevated phenylalanine with low tyrosine. The estimated age at which most patients with galactosemia were diagnosed ranged from 1 day to 5 months. Specifically, these times were estimated at 1-14 days for the UK, Italy, the Netherlands, the US (Boston, Salt Lake City, and Cincinnati), Germany, and Spain; 15-30 days for Mexico, Croatia, Argentina, and the Philippines, and 5.1 months for South Africa. In some instances isolated cases were diagnosed significantly later than the average due to atypically mild presentation or a false negative newborn screening result. Although in all cases GALT enzyme assays of hemolysates were used to confirm a diagnosis of classic galactosemia (13/13), some confirmation protocols described by our respondents also used GALT mutation analysis (7/13), total blood galactose (3/13), galactose-1-phosphate (gal-1P; 2/13), and/or urinary galactitol (2/13).

\section{Early intervention measures}

In all cases, respondents indicated that complete restriction of lactose intake was initiated immediately upon diagnosis (or suspected diagnosis) of an infant with classic galactosemia. All respondents agreed that galactosemic infants should not drink milk or milk-based formula, however, whether soy or elemental formula was recommended differed, as detailed below. Gastroenterology and ophthalmology evaluation was advised for symptomatic cases to enable treatment for possible liver dysfunction, sepsis and, occasionally, for cataracts.

The frequency of follow up visits for infants with classic galactosemia varied greatly, ranging from every two to three weeks for symptomatic cases to four times per year (see Table 2). Providers from Mexico, Italy, Spain, Argentina and Philippines performed monthly follow-ups mainly during the first three to six months and when solid food was first introduced. Providers from the Netherlands and Cincinnati in the US followed their patients as often as every 3 months so long as they remained free of acute symptoms. After the first year of life, the follow-up schedules varied by country and ranged from every three to four months within the first two years to once a year starting in mid-childhood (Table 2). In the case of South Africa, most patients were lost to follow-up in childhood.

As indicated in Table 3, the range of parameters assessed at follow-up in diagnosed infants varied between respondents, but could include assessment of liver (11/13) and renal functions (4/13), growth and development (13/13), ophthalmology evaluation (8/13), neurological assessment (3/13) including speech evaluation (2/13), measurement of red cell gal-1P (8/13), urine galactitol (3/13), and total blood galactose (3/13). Several respondents whose patients were diagnosed by newborn screening noted that they only checked liver and renal function at the first visit because these complications resolved so quickly following dietary restriction of galactose.

Following a confirmed diagnosis, galactosemic patients were usually referred to specialists (12/13) for subsequent follow-up, and these included: metabolic physicians (7/13), geneticists (2/13), or others (i.e., nutritionist or dietitian, ophthalmologist, gastroenterologist, 
speech therapist, psychologist, and neurologist). Of note, South African patients only had access to a general pediatrician.

As patients moved from infancy into childhood, disease management (Table 4) varied widely but generally included oversight of possible liver (4/12) or renal (1/12) dysfunction, endocrine complications (6/12), blood galactose/gal-1P and/or urinary galactitol levels $(9 / 12)$, growth parameters $(11 / 12)$, ophthalmology consults in the case of persistent cataracts (7/12), speech development and support (8/12), calcium homeostasis and bone mineral density (6/12), school performance (2/12) and neurological evaluation (2/12). The denominator in these fractions is 12 rather than 13 because we had no relevant information from one respondent.

\section{Dietary considerations}

All 13 respondents reported that infants diagnosed with classic galactosemia were put immediately on a galactose-restricted diet. A soy-based formula was the diet of choice in most of the cases (12/13) with the exception of Padova, Italy, where elemental, rather than soy, formula was recommended. In terms of solid foods, dietary restriction included avoiding dairy products in all cases - with some exceptions discussed below - but it also extended to include other more cryptic sources of galactose for some respondents, including those in Italy, Spain and the US (Cincinnati, OH and Salt Lake City, UT). In these locales dietary restriction included avoidance of some fruits and vegetables as well as other lactose or galactose-containing products. In the case of Cincinnati, $\mathrm{OH}$ the restriction only extended to those fruits and vegetables with high galactose content (i.e., figs, watermelon, legumes). In general, providers recommended that dietary restriction of galactose be maintained indefinitely (12/13); though a few providers (4/13) reported relaxing the stringency of dietary restriction for their older patients. In the case of Mexico, providers allowed consumption of 5-10 mg galactose/100 g of food after the first year of life. In Salt Lake City (US) the respondent reported allowing tomato-derived foods for patients 3 years of age and older, and aged cheeses for patients 6 years of age and older, with careful monitoring of gal-1 P levels to ensure there was no increase above baseline.

Most providers (11/13) reported that patients and families tended to adhere well to their recommended galactose-restricted diets, although providers from Mexico and Spain reported progressive loss of adherence as their pediatric patients matured. Factors such as cultural reliance on dairy in the diet (South Africa) and social stigma (Boston, US) were cited as potentially contributing to problems with dietary adherence.

\section{Puberty and teen years}

Of the 13 providers who responded to our survey, 10 had managed teenage patients with classic galactosemia. The recommended follow-up schedule for most of these providers (Table 2) was annual (9/10); for one it was twice per year. Important components of followup assessments during this period (Table 5) also varied between respondents and included: school performance $(8 / 10)$, behavior and social skills $(7 / 10)$, development and nutritional status (6/10), neurological development (5/10), ophthalmologic evaluation (4/10), liver function (3/10), and calcium homeostasis/bone density (7/10). Ovarian function in galactosemic girls was assessed by all respondents with female patients in this age range (9/9) through measurements of follicle stimulating hormone (FSH), luteinizing hormone (LH) and/or estradiol (8/9); some providers also supplemented these tests with a pelvic ultrasound (3/9). In some instances a fertility health specialist was consulted (3/9). Adherence to dietary galactose restriction, the current standard of care, was considered good in this age group (9/10). 


\section{Follow-up care for adult patients}

Of the eight respondents who managed adult patients, seven recommended annual follow-up (7/8); in Croatia adults were followed irregularly. Calcium homeostasis/bone density (8/8), female endocrine status and ovarian function (7/8), neurological parameters (5/8), and social skills (5/8) were listed as the most important follow-up components for this age group (see Table 6). Blood and urine markers of galactose accumulation (gal-1P, galactitol, total galactose) were followed by five of the eight respondents. School performance (4/8) and quality of life (2/8) were also monitored by some respondents. This long-term follow-up care in adults was overseen by a specialist in five out of eight cases.

Only respondents from the U.K. and US (Boston) had older adult patients ( $>50$ years old) in their care groups. For many of these older patients, neurological concerns were a principle reason for continued follow-up. The UK provider recommended that hormone replacement therapy (HRT) be discontinued in galactosemic women over age 50; the Boston provider recommended that HRT not be discontinued but reduced to avoid HRT-related risk complications.

\section{Frequency of long-term outcomes}

Figure 1 illustrates the combined percentages of patients reported by respondents as affected with individual long-term complications; the data were separated according to the average patient age at diagnosis for that respondent. The percentages plotted (and listed to the right of each bar) were generated by adding the individual numbers of affected vs. total patients evaluated for that outcome for the respondents in each group. Specific complications reported included: social or behavioral problems, cognitive impairment, ovarian insufficiency, neurological problems, reduced bone density, visual impairment, speech difficulties, weight/height issues, anemia, liver and/ or kidney disease and vitamin D deficiency. Of note, some outcome parameters were able to be evaluated in only a very limited number of patients for some groups ; the small sizes of these cohorts may contribute to the variance evident in some outcome frequencies. Further, some of these outcomes (e.g., liver dysfunction) were transient so that prevalence numbers fluctuated for some respondents. Countries from which information was ambiguous and outcome parameters for which fewer than three patients were evaluated (e.g., ovarian function among patients diagnosed after day 14 of life) were excluded from this figure.

\section{Discussion}

In this survey, we compared diagnostic and management practices for classic galactosemia in different populations around the world. We identified what were in some cases quite disparate approaches; these constituted a "natural experiment"; that might reveal the impact of environmental influence on outcomes in galactosemic individuals who were diagnosed at different times and/or clinically managed in different ways. Although newborn screening and presymptomatic intervention clearly reduce the acute morbidity and mortality of classic galactosemia (Schweitzer-Krantz 2003), some countries have decided to maintain clinical vigilance in lieu of pre-symptomatic screening for galactosemia because of the rarity of the disorder and the reality that clinical symptoms of galactosemia are often apparent before screening results can be reported in those countries (Honeyman et al 1993; SchweitzerKrantz 2003). In most countries that perform widespread newborn screening for galactosemia, which in our survey pool included the United States, Spain, the Netherlands, the Philippines, and Germany, diagnosis generally occurred within the first two weeks of life, though for some (Philippines) it occurred later. Early recognition was also the norm in Italy where diagnosis was made by a mix of newborn screening and clinical symptoms, and in the UK where the diagnosis was mostly made clinically, but where there is widespread 
clinical familiarity with galactosemia due to its relatively high incidence in this population (Fridovich-Keil and Walter 2008; Murphy et al 1999). In other areas, including Mexico, Croatia, Argentina, diagnosis occurred within a 15-30 day window, leaving affected babies exposed to the toxic effects of galactose for a longer time. This window was even longer in South Africa, where babies were not diagnosed until approximately 5 months of age. Unfortunately, in those populations in which galactosemia is diagnosed clinically, the diagnosis may be missed even in the presence of typical clinical symptoms (Schweitzer 1995; Schweitzer-Krantz 2003). Mexican providers who responded to our survey estimated that more than $80 \%$ of affected infants were missed in their country. In South Africa, a lack of clinical awareness of galactosemia appeared to be common (Henderson et al 2002), and most patients were lost to follow-up. The missed and delayed diagnoses in South Africa may in part reflect the milder disease phenotype associated with the S135L GALT mutation, which is predominant in the black South African population (Henderson et al 2002).

One might expect the long-term complications experienced by patients with galactosemia to be more common or more severe in those countries in which diagnosis was delayed; however, our survey responses did not support that hypothesis. Instead, our data supported the conclusion that while newborn screening for galactosemia saves lives, it does not change the frequency of long-term complications among those patients who survive; this same conclusion has been reported previously by others (Schweitzer-Krantz 2003). However, in countries that lack newborn screening for galactosemia, it is also possible that patients with atypical outcomes may simply escape diagnosis. It is further possible that intrinsic biological differences between populations - at the GALT locus or in the genetic background - may influence outcome, as has been suggested for African patients. To address these questions properly would require unbiased ascertainment through population-based screening for galactosemia worldwide, which is a laudable goal, but not a reality at this time.

\section{Prevalence}

The wide range of estimated disease frequency reported by our respondents $(1: 14,000$ to 1:80,000) also highlights the need for a more accurate assessment of the true frequency of galactosemia. Unexpectedly, some locales where diagnosis of classic galactosemia is made by newborn screening reported a lower prevalence (i.e., Salt Lake City in Utah, 1:60,000) than other locales where newborn screening for galactosemia has not been widely implemented (i.e., Buenos Aires in Argentina, 1:36,000). This range in responses may reflect true population differences in the frequency of galactosemia (e.g., (Honeyman et al 1993; Lee et al 2011), but it also may indicate that the estimates are incompatible, perhaps due to technical issues. For instance, differences in the approach or sensitivity of the methods used to detect GALT deficiency, or in defined cut-off levels in the screen, may leave some heterozygotes and/or Duarte variants included in some population estimates but not others (Kelly et al 1970). The population with the highest reported frequency of galactosemia in our study was South Africa, with an estimated prevalence of 1:14,000. This number was based on a population-based screen of cord blood samples for the S135L GALT mutation, which accounts for $>90 \%$ of classic galactosemia in this population (Henderson et al 2002). Epidemiologic studies involving full GALT gene sequencing in large populations would help to determine the true frequency of the gene defect, and this elucidation of the heterozygote carrier frequency would also be valuable for genetic counseling purposes (Robinson 1963).

\section{Disease monitoring}

A consensus is lacking as to the most relevant biomarker(s) to monitor in patients with classic galactosemia, and this was reflected in the survey responses we received. During the first few years of life, gal-1P was used as a follow-up marker by about half of the 
respondents (see Table 3). Indeed, gal-1P accumulation has long been regarded as the main factor accounting for the clinical severity of classic galactosemia (Pesce and Bodourian 1982), although no true causal link has been demonstrated, and the correlation between gal-1P levels and long-term disease outcome in patients with classic galactosemia has remained unclear (Hughes et al 2009; Schweitzer et al 1993; Walter et al 1999). Further, patients with Duarte galactosemia, who are believed to remain asymptomatic regardless of dietary galactose exposure, can demonstrate hemolysate gal-1P levels that overlap with those seen in untreated classic galactosemia patients (Ficicioglu et al 2008). Finally, a mouse model of GALT deficiency failed to mimic the patient phenotype despite accumulation of high levels of gal-1P after exposure to abundant dietary galactose (Leslie et al 1996; Ning et al 2000). Thus, frequent measurements of erythrocyte gal-1P for long-term monitoring in classic galactosemia may not be justified, although it can be argued that gal-1P levels should be monitored to track the impact of dietary modification, especially if the diet is being liberalized. In reality, there is limited capability for measuring gal-1P in some countries (for instance Croatia), further reducing its general efficacy as a monitoring parameter. Other than gal-1P, urinary galactitol was a commonly followed parameter cited by our respondents (see Table 3), despite the fact that its clinical significance also remains unclear (Walter et al 1999). Of note, unlike gal-1P, galactitol did not accumulate in the mouse model of classic galactosemia (Leslie et al 1996), which may suggest that the accumulation of both gal-1P and galactitol are required for phenotype expression.

\section{Treatment}

Dietary galactose restriction has been the mainstay in classic galactosemia treatment since 1935 (Mason and Turner 1935). Despite this long history, major controversies remain regarding the optimal strictness and duration of dietary galactose restriction (Bosch et al 2004a, b; Segal 1995). In this survey, all respondents advised their patients to restrict galactose and dairy intake upon diagnosis. This restriction was made by switching infants to an alternative formula, generally soy-based, although an elemental formula was prescribed in some cases. This recommendation was made based on the fact that elemental formula has even lower levels of galactose than soy formula, and initiation of elemental formula has been associated anecdotally with a significantly faster decrease of erythrocyte gal-1P in recently diagnosed infants with classic galactosemia (Ficicioglu et al 2005; Zlatunich and Packman 2005).

In agreement with a previous report (Bosch 2011), our findings indicate that as galactosemic patients grow from infancy to childhood, recommended dietary restrictions vary widely between health care providers. Whereas providers in some European countries (i.e., Italy and Spain) recommended a rigorous dietary galactose restriction that extends beyond dairy products to include all other sources of significant galactose including many fruits and vegetables, at least some providers in the UK, US, Germany and Mexico recommended more liberal dietary guidelines. Consistent with this approach, a recent follow-up study of five Estonian patients ages 7 to 14 years who had followed a lactose-restricted diet but had no limitations on eating mature cheeses, fruits, or vegetables reported outcomes that were consistent with those experienced by patients maintained on a more rigorously galactoserestricted diet (Krabbi et al 2011).

The conceptual basis for these more liberal recommendations derive from general agreement that fruits and vegetables provide nutritional benefit yet induce only a small increase in daily galactose intake (30-54 $\mathrm{mg}$ of galactose per day); this is minor relative to the estimated endogenous production of galactose in adult patients (1000 mg per day) (Berry et al 1995; Berry et al 2004; Huidekoper et al 2005). Indeed, it has been suggested that endogenous production of galactose may play a pivotal role in the pathogenesis of long-term complications in classic galactosemia (Berry et al 1995). Despite reports that suggest 
increased tolerance to exogenous galactose in adulthood (Lee et al 2003, Panis et al 2006a, b), which have led some to encourage a liberalization of galactose intake for this age group (Hughes et al 2009), others suggest it may be unwise to relax the diet in adulthood until the pathophysiology underlying the long-term complications in classic galactosemia is clearly understood (Bosch 2011).

\section{Long-term outcomes}

While most of our respondents had little to no experience managing older adult patients with galactosemia, many had at least some experience with patients as they moved through childhood and puberty into early adulthood. These respondents reported long-term outcomes and parameter frequencies that were consistent with those reported previously by others (Schweitzer-Krantz 2003; Waggoner et al 1990; Waisbren et al 2011), including primary or premature ovarian insufficiency in the vast majority of female patients, cognitive disability and/or behavioral/ social difficulties in at least half of the patients of most respondents, difficulties with speech in many patients, and neurological or movement difficulties in up to $40 \%$ of patients. One respondent noted that they saw speech difficulties more frequently in their pediatric patients than in their adult patients (Waisbren et al 2011) and wondered whether the adults might have developed effective coping mechanisms to minimize their speech difficulties, perhaps in response to speech therapy.

Primary ovarian insufficiency (POI) has long been a major cause of concern among women with classic galactosemia (Gibson 1995; Hoefnagel et al 1979; Kaufman et al 1979), greater than $80 \%$ of whom will experience POI despite treatment (Fridovich-Keil et al 2011). Early diagnosis and rigorous galactose restriction are not sufficient to prevent this long-term complication (Fridovich-Keil et al 2011; Gibson 1995; Waggoner et al 1990), and this conclusion was reinforced by our survey responses (Fig. 1). Of note, some of the outcome parameters illustrated in Fig. 1 appear to exhibit greater or lesser prevalence in patient groups diagnosed either earlier or later in life; considering the small sizes of some of these patient cohorts this may not be a meaningful difference.

Among our respondents, FSH, LH and/or estradiol were the main biomarkers used to follow ovarian function in galactosemic girls (see Table 5); some respondents measured these markers only in very young girls (e.g., 1-2 years old) or in older pre-teens (e.g., over 8 years old) when the hypothalamic-pituitary-ovarian axis should be active. Recent reports have suggested that anti-Müllerian hormone (AMH) may provide a meaningful predictor of ovarian status for pre-pubertal galactosemic girls across a broader age range (Fridovich-Keil et al 2011; Sanders et al 2009). None of our respondents in 2007 and 2008 reported monitoring AMH in their patients, though two (UK and Salt Lake City) noted that recently they have started to do so. Some survey respondents (3/9) also used pelvic ultrasound to assess their patients.

Currently, the mainstay of treatment for ovarian insufficiency in galactosemic girls and in the general population is hormone replacement therapy (HRT) (Fridovich-Keil et al 2011); most US providers initiate HRT between the ages of 12- to 14-years. In contrast, $40 \%$ of European clinicians initiate HRT by age 11 in their patients, and $~ 7 \%$ postpone this therapy until age 15 (Fridovich-Keil et al 2011; Kiess et al 2002). Our survey respondents also differed in their opinions regarding duration of HRT. To date, only indirect evidence suggests a potential benefit of long-term HRT in the prevention of endothelial dysfunction (Kalantaridou et al 2004) and osteoporosis (Anasti et al 1998). Of course, HRT does not alleviate the infertility experienced by many women with classic galactosemia (FridovichKeil et al 2011), although even women with reduced ovarian function may sometimes still achieve pregnancy (Gubbels et al 2009; Rubio-Gozalbo et al 2010). 
Cognitive disability (Antshel et al 2004; Bosch 2006; Kaufman et al 1995; Kaufman et al 1994; Manis et al 1997; Shield et al 2000), behavioral issues, and delays in the achievement of social milestones have been described for individuals with galactosemia (Bhat et al 2005; Bosch et al 2009; Bosch et al 2004a, b; Gubbels et al 2011). Some reports have suggested that the cognitive decline and speech difficulties that are highly prevalent in galactosemia may interfere with the development of social skills and educational attainment (Bosch et al 2009; Doyle et al 2010; Gubbels et al 2011). A lower health-related quality of life has also been reported among galactosemic patients; those aged 16 years and older reported significantly lower scores in the cognitive and social domains (Bosch et al 2004a, b). Thus, assessing a patient's quality of life is of value as it may allow incipient problems to be addressed at an earlier stage. That said, quality of life, career development, and social and educational issues were monitored by half or less than half of our respondents who followed adult patients (see Table 6).

Neurological complications, including tremor, have historically received less attention than many of the other complications, but this is beginning to change (Waisbren et al 2011). As more galactosemia patients survive to late childhood and adulthood worldwide, this outcome may be recognized as more common. One provider noted that some of his adult patients who presented with tremor in their teen years experienced an apparent progression of neurological symptoms for a number of years, after which time things "leveled off."

Interestingly, although calcium homeostasis and bone density are major components of follow-up care for pubertal and adult galactosemics (see Tables 5 and 6), none of our respondents noted frequent bone fractures or breaks as complications experienced by their patients. Of course, this may reflect the relatively young age of most of the patient cohorts. Annual supervision by a dietitian has long been recommended to ensure adequate nutrition and optimal calcium intake and thus to protect patients against osteoporosis, (Kaufman et al 1993; Walter et al 1999). Of note, abnormally low bone mineral density has been reported in both children and young adults with classic galactosemia (Kaufman et al 1993; Panis et al 2004; Rubio-Gozalbo et al 2002), however, this long-term complication may not simply reflect a nutritional deficiency. Previous reports correlate low bone density in galactosemics with defects in bone metabolism rather than nutritional deficiency (Panis et al 2004; RubioGozalbo et al 2002). A later report on the same study population showed that pre-pubertal but not pubertal children with galactosemia were meeting recommended dietary allowances for calcium but that children in both age groups increased their bone mineral density after calcium supplementation (Panis et al 2006a, b), leading the authors to conclude that calcium supplementation is useful in the management of galactosemia.

\section{Conclusions}

This report confirms that no current standard of care significantly ameliorates the high frequency of long-term complications in classic galactosemia. This conclusion is not new (Hughes et al 2009; Komrower 1982; Schweitzer-Krantz 2003; Waggoner et al 1990) but it is strengthened by the global breadth of the study reported here. Ovarian insufficiency, social, cognitive, and speech problems occurred in the majority of relevant cases reported here regardless of when treatment was begun, how stringently galactose intake was restricted, or how closely patients were monitored. Prior reports have suggested that some long-term clinical abnormalities experienced by patients with galactosemia may derive from fetal exposure to endogenously produced galactose or its metabolites in utero (Berry 2011; Holton and Leonard 1994; Schadewaldt et al 2010); however, attempts to minimize such exposure through maternal dietary intervention have failed to provide any measurable benefit (Holton and Leonard 1994). Although most of our respondents indicated they follow the same basic protocol for patients with galactosemia, including galactose restriction and 
monitoring gal-1P levels, our study highlights the current ambiguity in the management of patients with classic galactosemia worldwide and reinforces the need for further studies to increase our understanding of the long-term pathophysiology of this disorder, so that evidence-based best practice guidelines may eventually be established.

\section{Supplementary Material}

Refer to Web version on PubMed Central for supplementary material.

\section{Acknowledgments}

We thank all of the health care professionals and their staff who helped to collect the data reported in this manuscript. This work is dedicated with admiration to all galactosemia patients and their families.

Details of funding This work was supported by the National Institutes of Health grant R01 DK059904 (to JLFK).

\section{References}

Anasti J, Kalantaridou S, Kimzey L, Defensor R, Nelson L. Bone loss in young women with karyotypically normal spontaneous premature ovarian failure. Obstet Gynecol. 1998; 91:12-15. [PubMed: 9464713]

Antshel K, Epstein I, Waisbren S. Cognitive strengths and weaknesses in children and adolescents homozygous for the galactosemia Q188R mutation: a descriptive study. Neuropsychology. 2004; 18:658-664. [PubMed: 15506833]

Berry G. Is prenatal myo-inositol deficiency a mechanism of CNS injury in galactosemia? J Inherit Metab Dis. 2011; 34

Berry GT, Nissim I, Lin Z, Mazur AT, Gibson JB, Segal S. Endogenous synthesis of galactose in normal men and patients with hereditary galactosemia. Lancet. 1995; 346:1073-1074. [PubMed: 7564790]

Berry GT, Reynolds RA, Yager CT, Segal S. Extended [13C] galactose oxidation studies in patients with galactosemia. Mol Genet Metab. 2004; 82:130-136. [PubMed: 15172000]

Bhat M, Haase C, Lee P. Social outcome in treated individuals with inherited metabolic disorders: UK study. J Inherit Metab Dis. 2005; 28:825-830. [PubMed: 16435174]

Bosch AM. Classical galactosaemia revisited. J Inherit Metab Dis. 2006; 29:516-525. [PubMed: 16838075]

Bosch A. Classic galactosemia: dietary dilemmas. J Inherit Metab Dis. 2011; 34:257-260. [PubMed: 20625932]

Bosch AM, Bakker HD, Wenniger-Prick LJ, Wanders RJ, Wijburg FA. High tolerance for oral galactose in classical galactosaemia: dietary implications. Arch Dis Child. 2004a; 89:1034-1036. [PubMed: 15499058]

Bosch AM, Grootenhuis MA, Bakker HD, Heijmans HS, Wijburg FA, Last BF. Living with classical galactosemia: healthrelated quality of life consequences. Pediatrics. 2004b; 113:e423-e428. [PubMed: 15121984]

Bosch A, Maurice-Stam H, Wijburg F, Grootenhuis M. Remarkable differences: the course of life of young adults with galactosaemia and PKU. J Inherit Metab Dis. 2009; 32:706-712. [PubMed: 19816786]

Carney A, Sanders R, Garza K, et al. Origins, distribution and expression of the Duarte-2 (D2) allele of galactose-1-phosphate uridylyltransferase. Hum Mol Genet. 2009; 18:1624-1632. [PubMed: 19224951]

Doyle C, Channon S, Orlowska D, Lee P. The neuropsychological profile of galactosaemia. J Inherit Metab Dis. 2010; 33:603-609. [PubMed: 20607611]

Ficicioglu C, Yager C, Segal S. Galactitol and galactonate in red blood cells of children with the Duarte/galactosemia genotype. Mol Genet Metab. 2005; 84:152-159. [PubMed: 15670721] 
Ficicioglu C, Thomas N, Yager C, et al. Duarte (DG) galactosemia: a pilot study of biochemical and neurodevelopmental assessment in children detected by newborn screening. Mol Genet Metab. 2008; 95:206-212. [PubMed: 18976948]

Fridovich-Keil, JL.; Walter, JH. Galactosemia. In: Valle, D.; Beaudet, A.; Vogelstein, B.; Kinzler, K.; Antonarakis, S.; Ballabio, A., editors. The online metabolic \& molecular bases of inherited disease. McGraw-Hill; 2008. http://www.ommbid.com/

Fridovich-Keil J, Gubbels C, Spencer J, Sanders R, Land J, Rubio-Gozalbo E. Ovarian function in girls and women with GALT-deficiency galactosemia. J Inherit Metab Dis. 2011; 34:357-366. [PubMed: 20978943]

Gibson J. Gonadal function in galactosemics and in galactose-intoxicated animals. Eur J Pediatr. 1995; 154:S14-S20. [PubMed: 7671957]

Gubbels C, Kuppens S, Bakker J, et al. Pregnancy in classic galactosemia despite undetectable antiMüllerian hormone. Fertility and Sterility. Fertil Steril. 2009; 91(1293):e13-e16. [PubMed: 19200962]

Gubbels C, Maurice-Stam H, Berry G, et al. Psychosocial developmental milestones in men with classic galactosemia. J Inherit Metab Dis. 2011; 34:415-419. [PubMed: 21350966]

Henderson M, Shapiro L, McCowan C. Galactosemia detection from phenylketonuria screening. Clin Chem. 1988; 34:188-189. [PubMed: 3338165]

Henderson H, Leisegang F, Brown R, Eley B. The clinical and molecular spectrum of galactosemia in patients from the Cape Town region of South Africa. BMC Pediatr. 2002; 2:7. [PubMed: 12350230]

Hoefnagel D, Wurster-Hill D, Child E. Ovarian failure in Galactosaemia. Lancet. 1979; 314:1197. [PubMed: 91932]

Holton JB, Leonard JV. Clouds still gathering over galactosaemia. Lancet. 1994; 344:1242-1243. [PubMed: 7526095]

Honeyman M, Green A, Holton J, Leonard J. Galactosaemia: results of the British Paediatric Surveillance Unit Study, 1988-90. Arch Dis Child. 1993; 69:339-341. [PubMed: 8215542]

Hughes J, Ryan S, Lambert D, et al. Outcomes of siblings with classical galactosemia. J Pediatr. 2009; 154:721-726. [PubMed: 19181333]

Huidekoper H, Bosch A, van der Crabben S, Sauerwein H, Ackermans M, Wijburg F. Short-term exogenous galactose supplementation does not influence rate of appearance of galactose in patients with classical galactosemia. Mol Genet Metab. 2005; 84:265-272. [PubMed: 15694176]

Kalantaridou S, Naka K, Papanikolaou E, et al. Impaired endothelial function in young women with premature ovarian failure: normalization with hormone therapy. J Clin Endocrinol Metab. 2004; 89:3907-3913. [PubMed: 15292326]

Kaufman F, Kogut M, Donnell G, Koch H, Goebelsmann U. Ovarian failure in galactosaemia. Lancet. 1979; 2:737-738. [PubMed: 90818]

Kaufman FR, Loro ML, Azen C, Wenz E, Gilsanz V. Effect of hypogonadism and deficient calcium intake on bone density in patients with galactosemia. J Pediatr. 1993; 123:365-370. [PubMed: 8355111]

Kaufman FR, Reichardt JK, Ng WG, et al. Correlation of cognitive, neurologic, and ovarian outcome with the Q188R mutation of the galactose-1-phosphate uridyltransferase gene. J Pediatr. 1994; 125:225-227. [PubMed: 8040766]

Kaufman FR, McBride-Chang C, Manis FR, Wolff JA, Nelson MD. Cognitive functioning, neurologic status and brain imaging in classical galactosemia. Eur J Pediatr. 1995; 154:S2-S5. [PubMed: 7671958]

Kelly S, Katz S, Burns J, Boylan J. Screening for galactosemia in New York State. Public Health Rep. 1970; 85:575-578. [PubMed: 4987471]

Kiess W, Conway G, Ritzen M, et al. Induction of puberty in the hypogonadal girl-practices and attitudes of pediatric endocrinologists in Europe. Horm Res. 2002; 57:66-71. [PubMed: 12006724]

Komrower G. Galactosaemia - Thirty years on. The experience of a generation. F.P. Hudson Memorial Lecture. J Inherit Metab Dis. 1982; 5:96-104. 
Krabbi K, Uudelepp M, Joost K, Zordania R, Õnap K. Long-term complications in Estonian galactosemia patients with a less strict lactose-free diet and metabolic control. MolGenet Metab. 2011; 103:249-253.

Lee PJ, Lilburn M, Wendel U, Schadewaldt P. A woman with untreated galactosaemia. Lancet. 2003; 362:446. [PubMed: 12927432]

Lee B, Cheon C, Kim J, et al. Low prevalence of classical galactosemia in Korean population. J Hum Genet. 2011; 56:94-96. [PubMed: 21150919]

Leslie ND, Yager KL, McNamara PD, Segal S. A mouse model of galactose-1-phosphate uridyl transferase deficiency. Biochem Mol Med. 1996; 59:7-12. [PubMed: 8902187]

Manis F, Cohn L, McBride-Chang C, Wolff J, Kaufman F. A longitudinal study of cognitive functioning in patients with classical galactosaemia, including a cohort treated with oral uridine. $\mathrm{J}$ Inherit Metab Dis. 1997; 20:549-555. [PubMed: 9266391]

Mason HH, Turner ME. Chronic galactosemia. Am J Dis Child. 1935; 50:359-376.

Murphy M, McHugh B, Tighe O, et al. Genetic basis of transferase-deficient galactosaemia in Ireland and the population history of the Irish Travellers. Eur J Hum Genet. 1999; 7:549-554. [PubMed: 10439960]

Ning C, Reynolds R, Chen J, et al. Galactose metabolism by the mouse with galactose-1-phosphate uridyltransferase deficiency. Pediatr Res. 2000; 48:211-217. [PubMed: 10926297]

Panis B, Forget PP, van Kroonenburgh MJPG, et al. Bone metabolism in galactosemia. Bone. 2004; 35:982-987. [PubMed: 15454106]

Panis B, Bakker JA, Sels J-PJE, Spaapen LJM, van Loon LJC, Rubio-Gozalbo ME. Untreated classical galactosemia patient with mild phenotype. Mol Genet Metab. 2006a; 89:277-279. [PubMed: 16621642]

Panis B, Vermeer C, van Kroonenburgh MJPG, et al. Effect of calcium, vitamins K1 and D3 on bone in galactosemia. Bone. 2006b; 39:1123-1129. [PubMed: 16782422]

Pesce M, Bodourian S. Clinical significance of plasma galactose and erythrocyte galactose-1phosphate measurements in transferase-deficient galactosemia and in individuals with belownormal transferase activity. Clin Chem. 1982; 28:301-305. [PubMed: 6276048]

Robinson A. The assay of galactokinase and galactose-1-phosphate uridyl transferase activity in human erythrocytes. A presumed test for heterozygous carriers of the galactosemic defect. J Exp Med. 1963; 118:359-370.

Rubio-Gozalbo M, Hamming S, van Kroonenburgh M, Bakker J, Vermeer C, Forget P. Bone mineral density in patients with classic galactosaemia. Arch Dis Child. 2002; 87:57-60. [PubMed: 12089126]

Rubio-Gozalbo M, Gubbels C, Bakker J, Menheere P, Wodzig W, Land J. Gonadal function in male and female patients with classic galactosemia. Hum Reprod Update. 2010; 16:177-188. [PubMed: 19793842]

Sanders R, Spencer J, Epstein M, et al. Biomarkers of ovarian function in girls and women with classic galactosemia. Fertil Steril. 2009; 92:344-351. [PubMed: 18684449]

Schadewaldt P, Hoffmann B, Hammen H, Kamp G, Schweitzer-Krantz S, Wendel U. Longitudinal assessment of intellectual achievement in patients with classical galactosemia. Pediatrics. 2010; 125:e374-e381. [PubMed: 20100763]

Schweitzer S. Newborn mass screening for galactosemia. Eur J Pediatr. 1995; 154:S37-S39. [PubMed: 7671962]

Schweitzer S, Shin Y, Jakobs C, Brodehl J. Long-term outcome in 134 patients with galactosemia. Eur J Pediatr. 1993; 152:36-43. [PubMed: 8444204]

Schweitzer-Krantz S. Early diagnosis of inherited metabolic disorders towards improving outcome: the controversial issue of galactosaemia. Eur J Pediatr. 2003; 162:S50-S53. [PubMed: 14614623]

Segal S. Galactosemia unsolved. Eur J Pediatr. 1995; 154:S97-S102. [PubMed: 7671976]

Shield JP, Wadsworth EJ, MacDonald A, et al. The relationship of genotype to cognitive outcome in galactosaemia. Arch Dis Child. 2000; 83:248-250. [PubMed: 10952646]

Waggoner DD, Buist NR, Donnell GN. Long-term prognosis in galactosaemia: results of a survey of 350 cases. J Inherit Metab Dis. 1990; 13:802-818. [PubMed: 1706789] 
Waisbren S, Potter N, Gordon C, et al. The adult galactosemic phenotype. J Inherit Metab Dis. 2011; 35:279-286. [PubMed: 21779791]

Walter JH, Collins JE, Leonard JV, Chiswick M, Marcovitch H. Recommendations for the management of galactosaemia • Commentary. Arch Dis Child. 1999; 80:93-96. [PubMed: 10325771]

Zlatunich C, Packman S. Galactosaemia: early treatment with an elemental formula. J Inherit Metab Dis. 2005; 28:163-168. [PubMed: 15877205] 


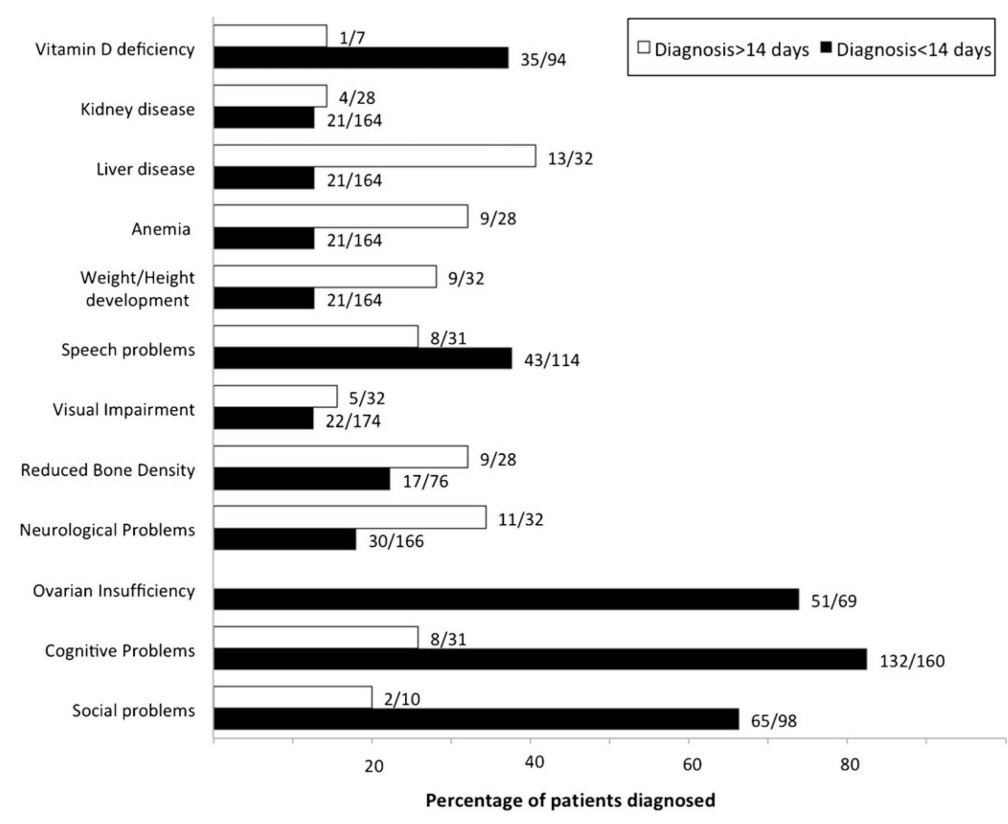

Fig. 1.

Long-term outcomes among patient cohorts grouped by average age at diagnosis. White bars represent data from respondents whose patients with classic galactosemia were diagnosed predominantly before 14 days of life (Netherlands, UK, Italy, and US (Cincinnatti, OH and Boston, MA). Shaded bars represent data from respondents whose patients with classic galactosemia were diagnosed predominantly after day 14 of life (Croatia, Mexico, and Philippines). Other respondents were not included in this figure due to lack of information. Some outcome parameters were evaluated in only a very limited number of patients for some groups, and data are not presented for ovarian function among patients diagnosed after day 14 of life because there were $<3$ patients in this group. The numbers of patients included in Table 1 do not necessarily match those presented in Fig. 1 as not all patients were able to be evaluated for all long-term outcomes. Data graphed in Fig. 1 include only those patients for whom relevant outcome information was available 
Table 1

Racial distribution of patient population reported by survey respondents (total=234 patients)

\begin{tabular}{|c|c|c|c|c|c|c|}
\hline Country (City) & Total & $\begin{array}{l}\text { African- } \\
\text { American }\end{array}$ & African & Asian & Caucasian & Hispanic \\
\hline UK (Manchester) & 56 & 2 & 0 & 3 & 51 & 0 \\
\hline Netherlands (Amsterdam) & 17 & 1 & 0 & 0 & 16 & 0 \\
\hline Mexico (Mexico, D.F.) & 21 & 0 & 0 & 0 & 0 & 21 \\
\hline Croatia (Zagreb) & 7 & 0 & 0 & 0 & 7 & 0 \\
\hline Italy (Padova) & 18 & 0 & 0 & 1 & 17 & 0 \\
\hline $\begin{array}{l}\text { Spain (Santiago de } \\
\text { Compostela) }\end{array}$ & 2 & 0 & 0 & 0 & 2 & 0 \\
\hline Germany (Düsseldorf) & 10 & 0 & 0 & 0 & 9 & 1 \\
\hline South Africa (Cape Town) & 1 per year ${ }^{*}$ & 0 & 1 & 0 & 0 & 0 \\
\hline United States (Cincinnati) & No information & & & & & \\
\hline $\begin{array}{l}\text { United States (Salt Lake } \\
\text { City) }\end{array}$ & 22 & 0 & 0 & 0 & 22 & 0 \\
\hline United States (Boston) & 72 & 2 & 0 & 0 & 67 & 3 \\
\hline Argentina (Buenos Aires) & 4 & 0 & 0 & 0 & 0 & 4 \\
\hline Manila (Philippines) & 4 & 0 & 0 & 4 & 0 & 0 \\
\hline
\end{tabular}


Table 2

Frequency of monitoring during infancy and childhood. Follow-up may be more frequent if acute symptoms persist

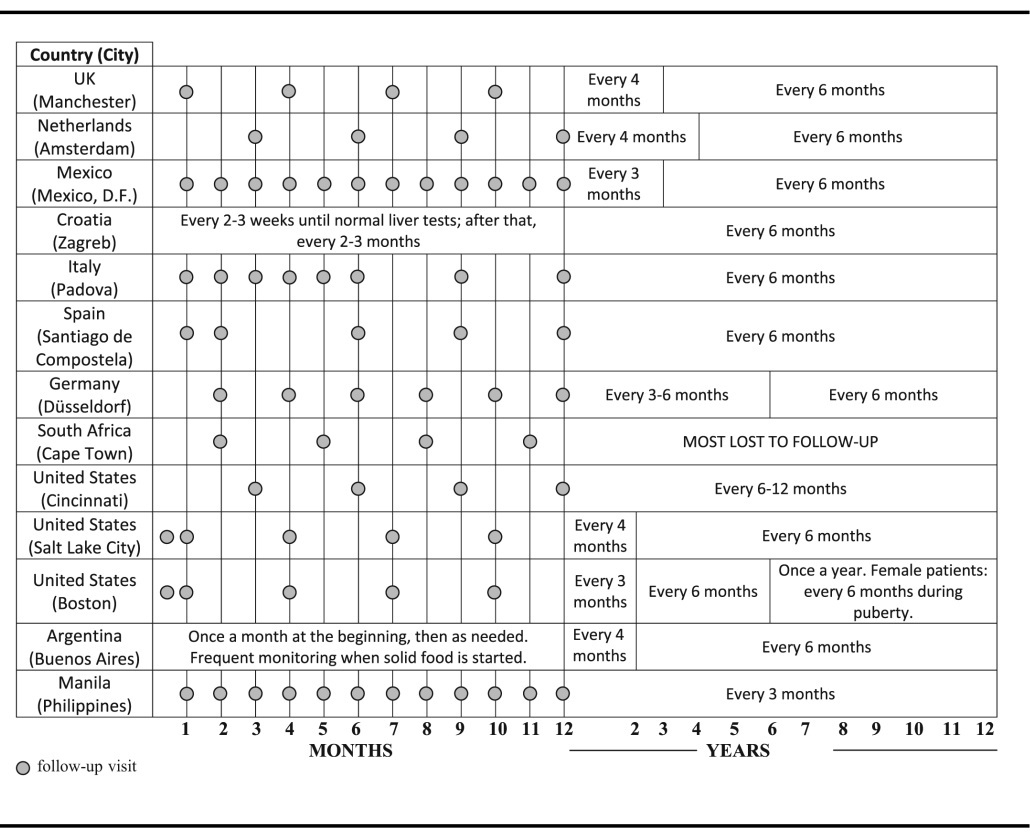




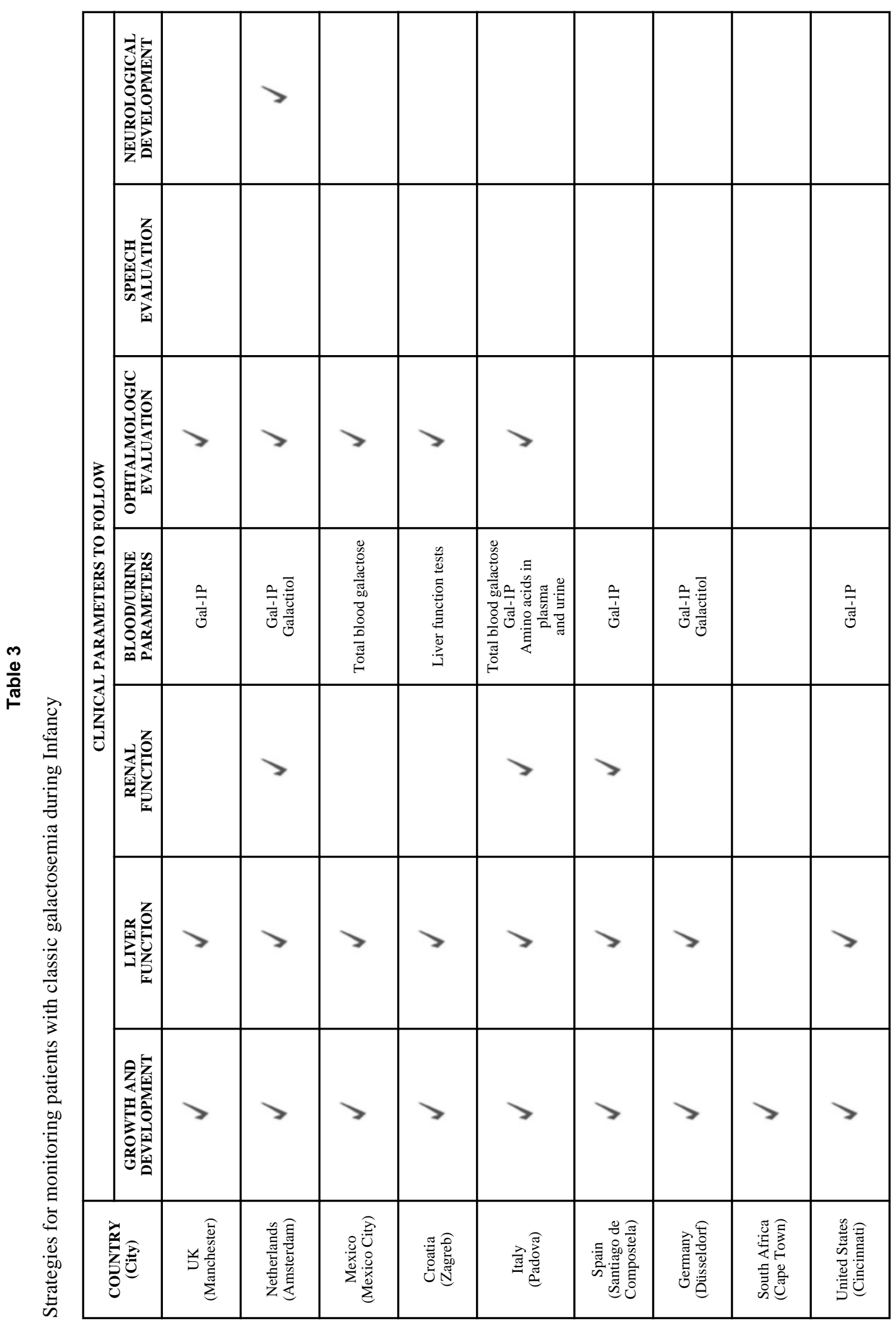

J Inherit Metab Dis. Author manuscript; available in PMC 2013 November 01. 


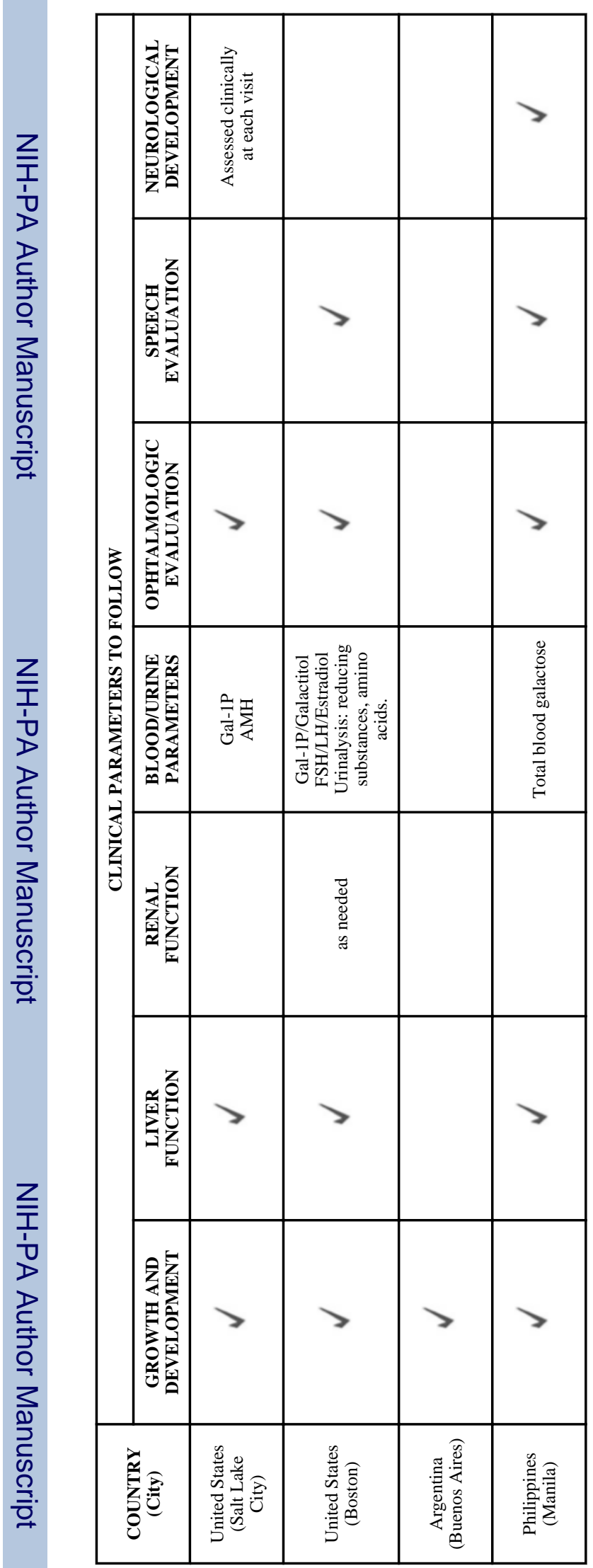

J Inherit Metab Dis. Author manuscript; available in PMC 2013 November 01. 


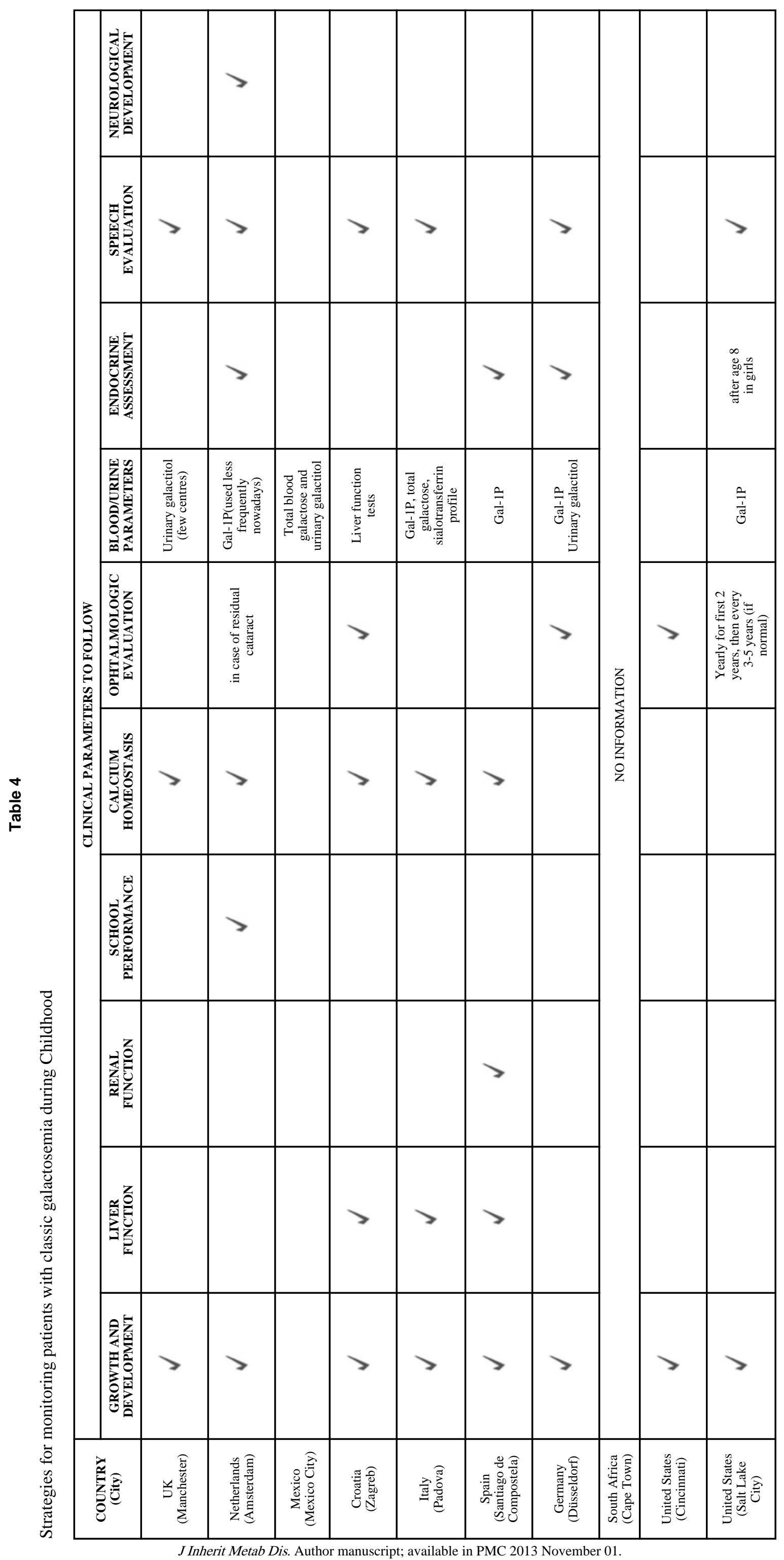

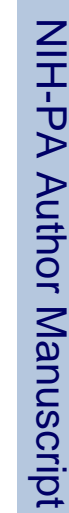

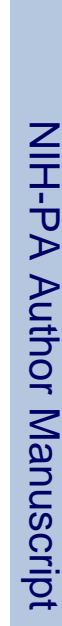

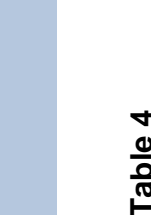

吾 


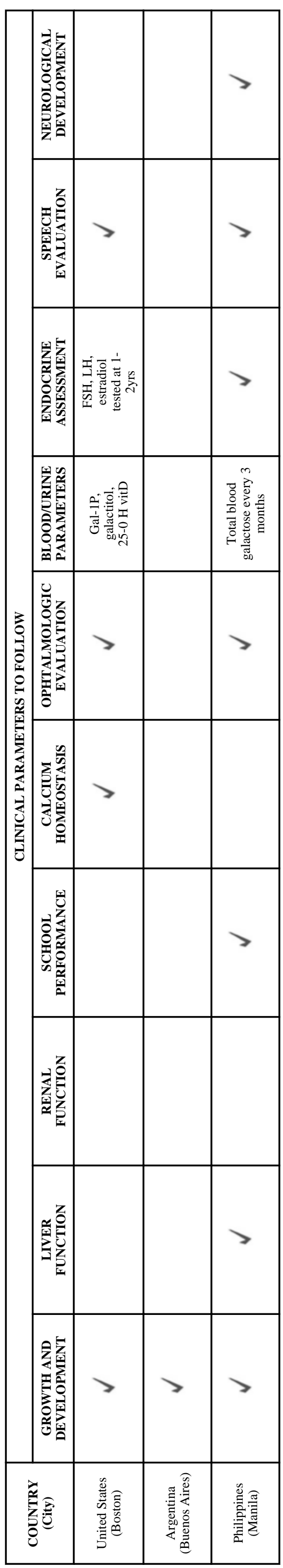




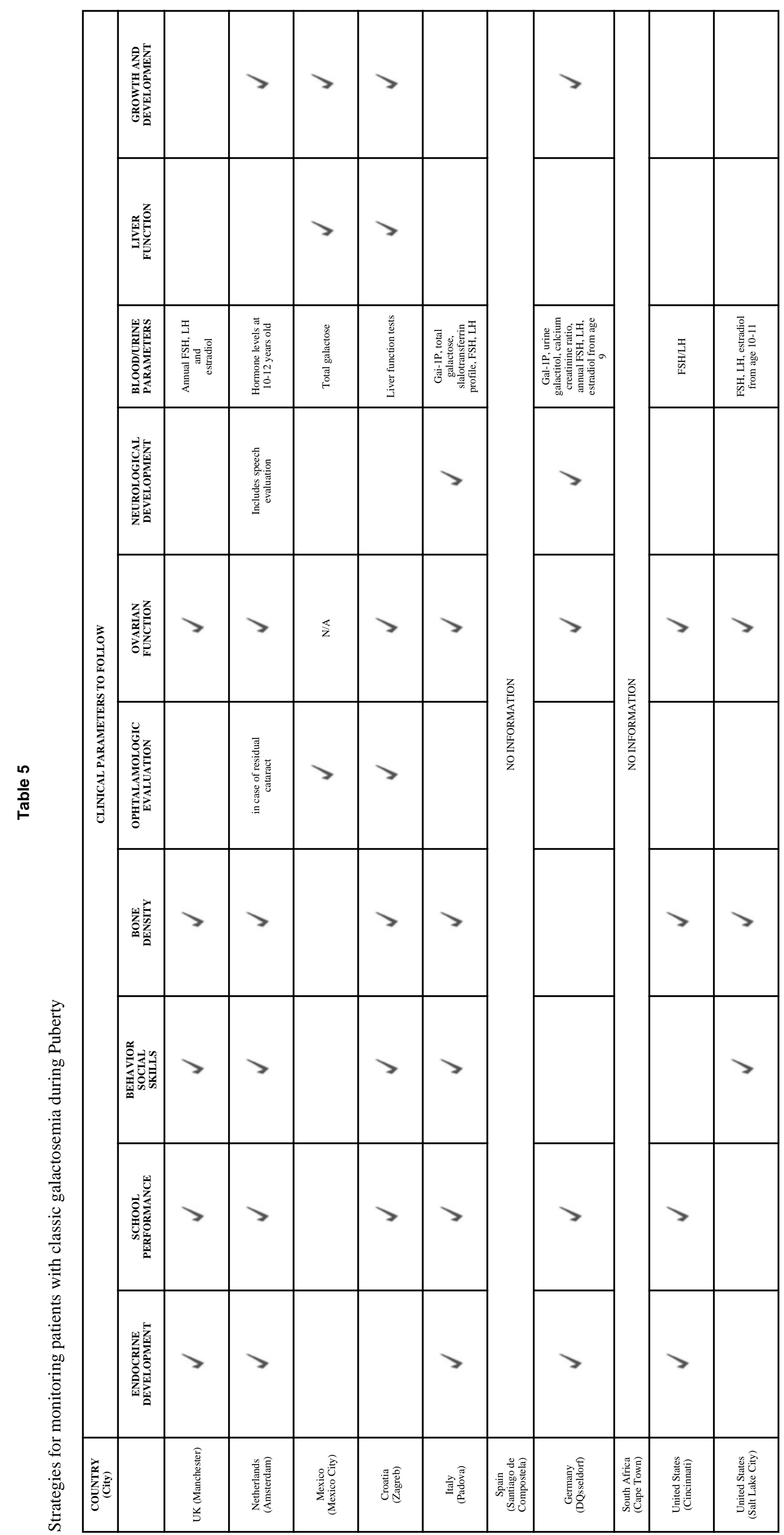

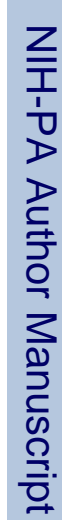

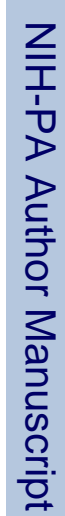

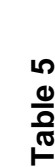

J Inherit Metab Dis. Author manuscript; available in PMC 2013 November 01. 


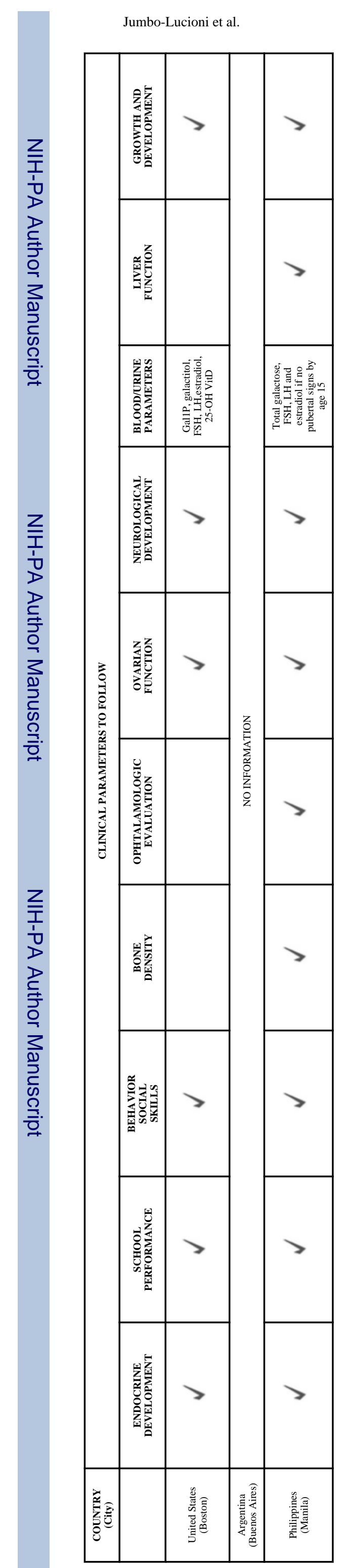

Page 24

J Inherit Metab Dis. Author manuscript; available in PMC 2013 November 01. 


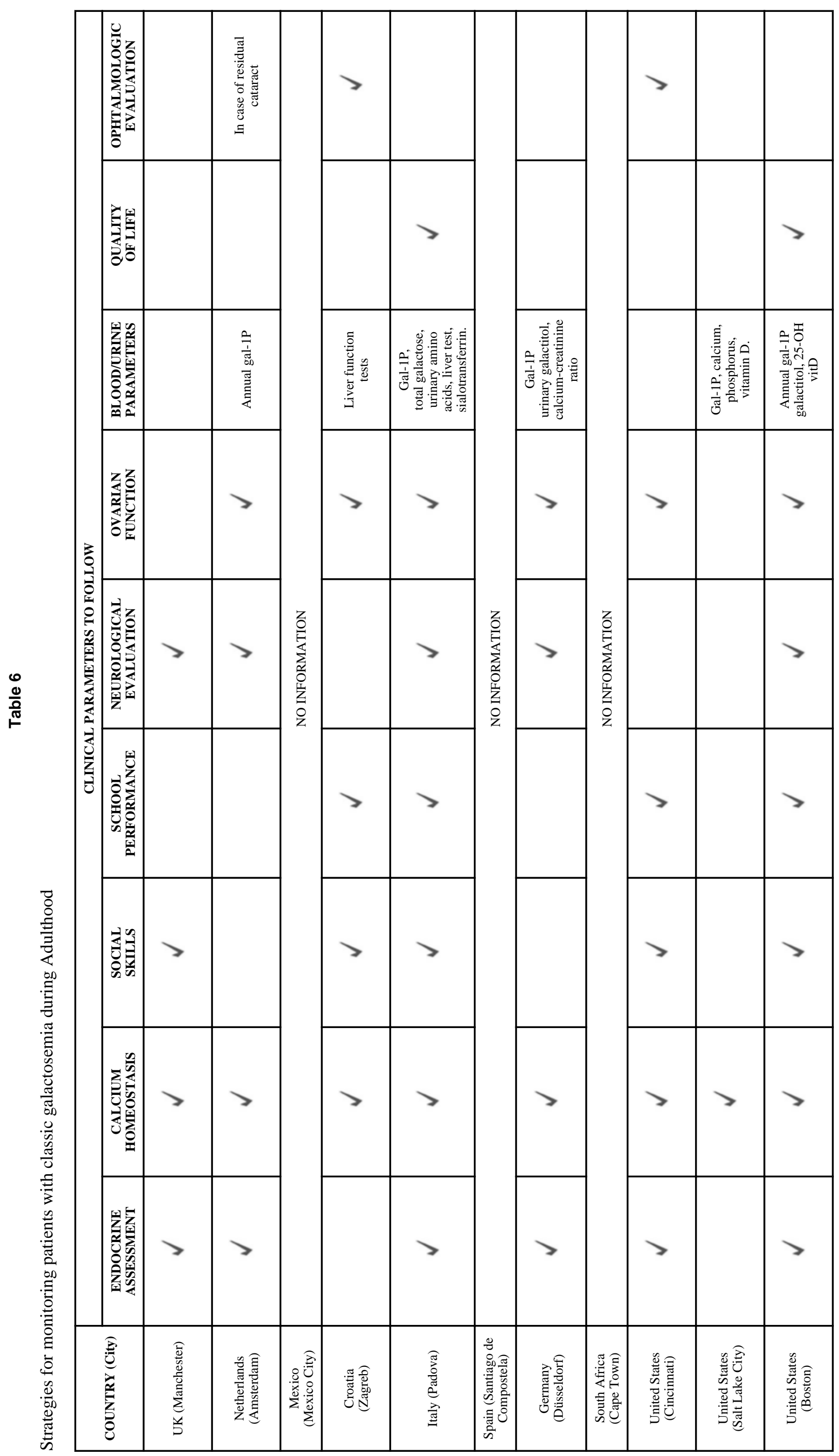

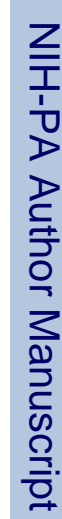

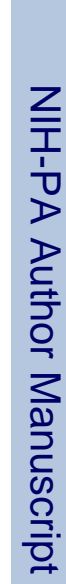

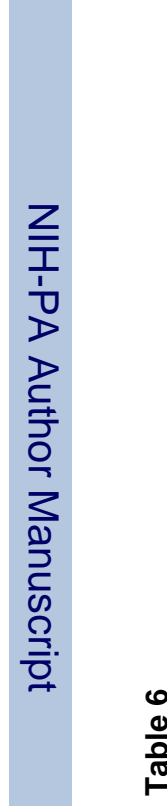




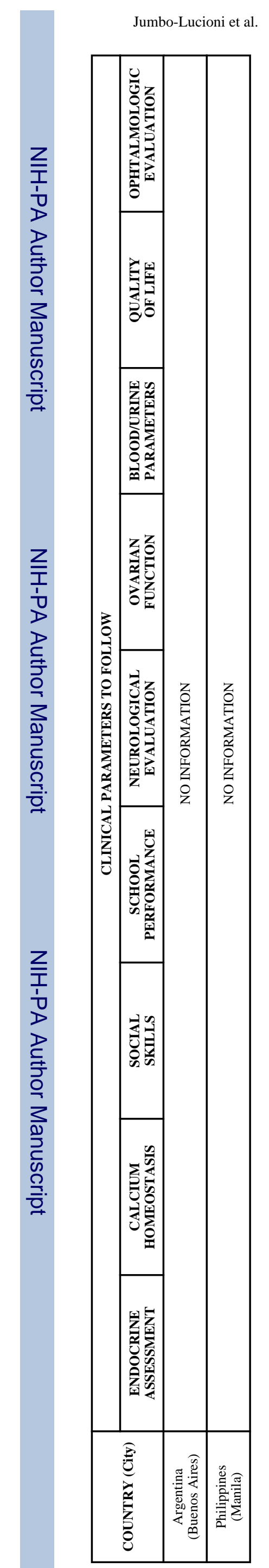

Page 26 